\title{
Databases and tools for constructing signal transduction networks in cancer
}

\author{
Seungyoon $\mathrm{Nam}^{1,2,3, *}$ \\ ${ }^{1}$ Department of Life Sciences, Gachon University, Seongnam 13120, ${ }^{2}$ Department of Genome Medicine and Science, College of Medicine, \\ Gachon University, ${ }^{3}$ Gachon Institute of Genome Medicine and Science, Gachon University Gil Medical Center, Incheon 21565, Korea
}

Traditionally, biologists have devoted their careers to studying individual biological entities of their own interest, partly due to lack of available data regarding that entity. Large, highthroughput data, too complex for conventional processing methods (i.e., "big data"), has accumulated in cancer biology, which is freely available in public data repositories. Such challenges urge biologists to inspect their biological entities of interest using novel approaches, firstly including repository data retrieval. Essentially, these revolutionary changes demand new interpretations of huge datasets at a systems-level, by so called "systems biology". One of the representative applications of systems biology is to generate a biological network from high-throughput big data, providing a global map of molecular events associated with specific phenotype changes. In this review, we introduce the repositories of cancer big data and cutting-edge systems biology tools for network generation, and improved identification of therapeutic targets. [BMB Reports 2017; 50(1): 12-19]

\section{INTRODUCTION}

Traditionally, researchers have focused their efforts on single biological phenomena (e.g., a single gene mutation) or a specific signaling pathway (1). Now, the age of "omics" big data has brought about cutting-edge processing methods for interpreting biological mega data, which have now universally adopted. Based on such mega data (so-called "big data"), researchers aim to understand systems-level-based phenotype changes $(1,2)$ by assessing entire pathways/networks, and not just a single entity. Systems biology is defined as a framework (3) to enable systems-level understanding for generating new biological hypotheses, by computational modeling of massive

${ }^{*}$ Corresponding author. Tel: +82-32-460-2179; Fax: +82-32-4602365; E-mail: nams@gachon.ac.kr

https://doi.org/10.5483/BMBRep.2017.50.1.135

Received 8 August 2016

Keywords: Cancer, Network biology, Signaling network, Systems biology high-throughput data.

Currently, systems biology has broadened its applications from basic science (including small RNAs) (4-6) toward translational medicine, including biomarker and therapeutic target identification $(1-3,7,8)$. Systems biology often begins from high-throughput experimental data. Due to mammoth data deposition, as well as data generation by various next-generation sequencing (NGS) techniques (9), big data science has emerged, in particular, from the field of cancer genomics (10). The most widely used repositories include The Cancer Genome Atlas (TCGA) Research Network (11) and the International Cancer Genome Consortium (ICGC) (12). The development of applications for big data science (10) has been facilitated by systems biology frameworks to allow interpretation of systems-level tumorigenesis and molecular mechanisms.

Systems biology covers several diverse areas (13): hypothesis generation and network construction (or inference), and network simulation (e.g., ordinary differential equations, boolean dynamics). In this review, we restrict our discussion to network generation, while also describing analysis tools and relating databases in the field of cancer.

\section{A WORKFLOW OF SYSTEMS BIOLOGY}

Systems biology has a straightforward workflow of components (13), as shown in Fig. 1A. To understand systems-level biology, observations for all entries are necessary, and highthroughput data is merely a starting point. Computational modeling takes the high-throughput data and, in certain circumstances, prior knowledge (including pathways and gene sets) is selected, resulting in network inference and hypothesis generation (13). Depending on whether computational modeling is used with or without prior knowledge, one may employ both data-driven network modeling and hybrid network modeling, respectively (14). In both of them, computational modeling is a key component, due to its ability to deal with the complexity of interconnectivity among systems entries $(13,14)$.

\section{HIGH-THROUGHPUT DATA AND ITS REPOSITORIES}

Currently, there are numerous types of high-throughput data (i.e., "omics"), including genomics, epigenomics, transcriptomics, 
A.

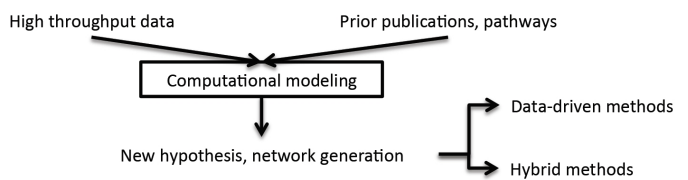

B.

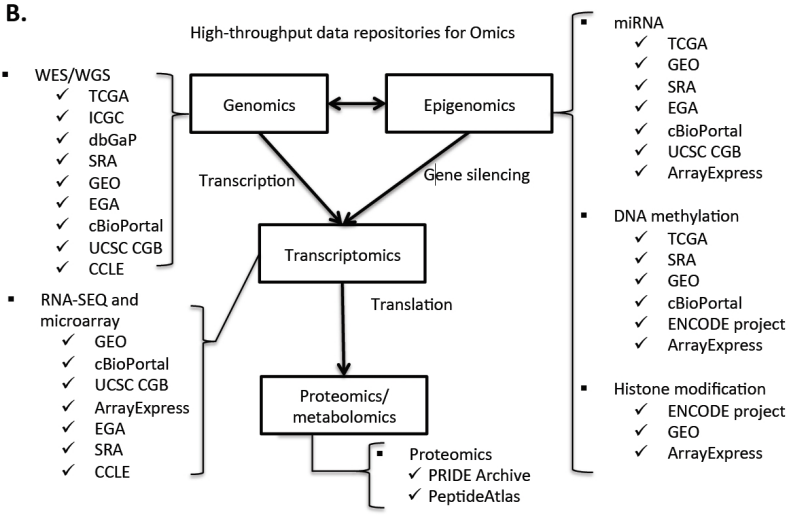

C.

Edge connections in ARACNE, and WGCNA
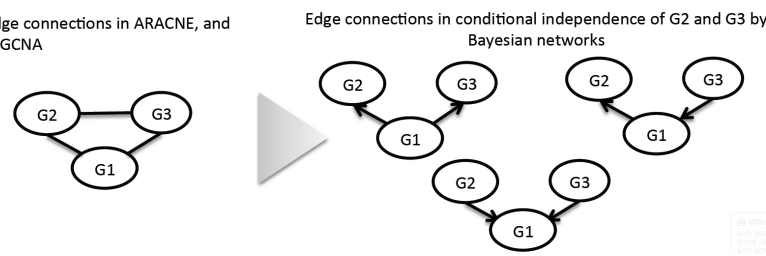

Fig. 1. Systems biology, databases, and network generation. (A) The diversity of types of high-throughput data (genomics, epigenomics, transcriptomics, proteomics, metabolomics) available. The relationships among the data types are connected by edges. (B) The flow (represented by "edges") of genetic information from DNA to protein is aligned with the diverse data types. Public repositories corresponding to each data type are listed (further description in Table 1). (C) Network differences between correlationbased approaches and Bayesian networks approaches. The correlation (or mutual information) oriented tools, ARACNE (39) and WGCNA (36), do not report directions of edges in networks. Bayesian-driven networks naturally reveal directed edges among the network entries. In other words, the undirected network (in left of the grey-shaded triangular) having G1, G2, and G3 entries by ARACNE and WGCNA can be differentiated into directed networks (in the right of the grey-shaded triangular), using Bayesian networks tools (48-51).

metabolomics, and proteomics (15). As shown in Fig. 1B, the omics data types are aligned with the flow of genetic information in biology. Cancer genomics data in various types of cancers, including whole genome sequencing (WGS), whole exome sequencing (WES), and SNP array, has already been deposited in several public repositories including The Cancer Genome Atlas (TCGA) (11), and International Cancer Genome Consortium (ICGC) $(12,16)$ (Fig. 1B). Epigenomics in public databases, including the Encyclopedia of DNA Elements (ENCODE) (17) and the Database of Genotypes and Phenotypes (dbGaP) (18), possess next-generation sequencing datasets for genome-wide DNA methylation, histone modifications, transcription factor binding, and non-coding RNAs (e.g., miRNAs, piRNAs). Transcriptomic datasets are deposited in the Gene Expression Omnibus (GEO) (19), and ArrayExpress (20), for more than 10 years. Proteomics and metabolomics have now begun accumulation in the PeptieAtlas (21) and the PRoteomics IDEntifications (PRIDE) (22) databases. Each repository in Fig. $1 \mathrm{~A}$ is not restricted to one specific data type, and users should be prudent to inspect all the data types of their interest through multiple repositories, and not a single one. The brief information of the repositories is described in Table 1.

\section{PRIOR KNOWLEDGE}

The two representative categories in prior knowledge are gene sets and pathway databases (including protein-protein interactions). A gene set consists of the relevant biological description and its gene entries. The MIT MSigDB Collections (23) (software.broadinstitute.org/gsea/msigdb/collections.jsp), one of most comprehensive repositories of gene sets, contains 13,311 entries. Recently, gene sets have begun including miRNA genes (and their expression), as well as protein-coding genes (24). By definition, however, gene sets do not contain hierarchy or mutual interaction for their gene entries (25). To accommodate such non-hierarchy, gene sets have been mainly applied to various enrichment analyses that utilize KolmogorovSmirnov test statistic, ANOVA, or hypergeometric test (further review in $(26,27))$. A recent approach (28) identifies the conditional dependency in a gene set, to reconstruct hierarchical relationships. Thus, numerous gene sets have now been recognized as prior knowledge for use in network generation.

Unlike gene sets, pathways or protein-protein interactions have hierarchy or mutual relationships among the entries. Of numerous, diverse pathway databases, we describe the Kyoto Encyclopedia of Genes and Genomes (KEGG) (29), Reactome (30), STRING (31), and human-integrated pathway (hiPathDB) (32) databases. In particular, the KEGG (29) pathway database, one of the popular manually-curated pathway resources, consists of seven types of network contexts: cellular processes, metabolism, genetic information processing, environmental information processing, human diseases, organismal systems, and drug development (29). The KEGG pathway information is machine-readable via KGML (KEGG Markup Language). Reactome (30) is another popular peer reviewed pathway database, and contains $>6,700$ reactions (e.g., phosphorylation, acetylation, etc.) extracted from 15,000 publications. For machine readability, the SBML (Systems Biology Markup Language) version of Reactome data is also available (33).

The database and web resource STRING (Search Tool for the Retrieval of Interacting Genes/Proteins, string-db.org) contains a very extensive collection of protein-protein interactions, based on publications and predictions. The interaction entries of STRING (31) amount to 932,553,897, from 2,031 organisms (as of 2016-04-19). While KEGG and Reactome both have 
Table 1. Cancer-related, high-throughput data repositories. The databases in Fig. 1B are described with additional information including the number of available data sets, data types, and websites. The number of entries is deemed valid as of 05/02/2016

\begin{tabular}{|c|c|c|c|}
\hline Names & Description & Address & Cancer relating data \\
\hline TCGA & $\begin{array}{l}\text { The Cancer Genome Atlas (TCGA): now one of programs organized } \\
\text { by newly established NCl's Center for Cancer Genomics (11) }\end{array}$ & $\begin{array}{l}\text { cancergenome. } \\
\text { nih.gov }\end{array}$ & $\begin{array}{l}34 \text { cancer studies (types), } \\
11,091 \text { samples }\end{array}$ \\
\hline $\mathrm{dbGaP}$ & $\begin{array}{l}\text { The database of Genotypes and Phenotypes (dbGaP): archive of } \\
\text { genome and phenotype in human }\end{array}$ & $\begin{array}{l}\text { www.ncbi.nlm. } \\
\text { nih.gov/gap }\end{array}$ & 991 datasets \\
\hline SRA & $\begin{array}{l}\text { Sequence Read Archive (SRA): raw sequencing files and alignment } \\
\text { files from next generation sequencing }\end{array}$ & $\begin{array}{l}\text { www.ncbi.nlm. } \\
\text { nih.gov/sra }\end{array}$ & 1,950 cancer studies \\
\hline cBioPortal & $\begin{array}{l}\text { Multi-functional platform: supporting intuitive visualization, literate clinical } \\
\text { pie chart, and simple data access (75). TCGA data visualization included. }\end{array}$ & cbioportal.org & $\begin{array}{l}126 \text { cancer genomics } \\
\text { studies, } 26,080 \text { samples }\end{array}$ \\
\hline ICGC & $\begin{array}{l}\text { The International Cancer Genome Consortium (ICGC): } \\
\text { global-scale cancer projects (16) }\end{array}$ & dcc.icgc.org/ & $\begin{array}{l}66 \text { cancer projects, } \\
17,867 \text { donors }\end{array}$ \\
\hline ArrayExpress & An archive of functional genomics data (76) & $\begin{array}{l}\text { www.ebi.ac.uk/ } \\
\text { arrayexpress }\end{array}$ & 14,974 datasets \\
\hline EGA & The European Genome-phenome Archive (EGA) & $\begin{array}{l}\text { www.ebi.ac.uk/ } \\
\text { ega/home }\end{array}$ & 1,997 datasets \\
\hline UCSC CGB & $\begin{array}{l}\text { UCSC Cancer Genomics Browser (UCSC CGB): supplying interactive } \\
\text { heat-map based visualization, and ready-to-use tab-delimited genomics } \\
\text { and clinical data download (77). TCGA data visualization included. }\end{array}$ & $\begin{array}{l}\text { genome-cancer. } \\
\text { ucsc.edu }\end{array}$ & 720 datasets \\
\hline GEO & $\begin{array}{l}\text { The Gene Expression Omnibus (GEO) (19): a public repository for } \\
\text { microarray and next-generation sequencing data sets, and one } \\
\text { of the representative repositories. }\end{array}$ & $\begin{array}{l}\text { www.ncbi.nlm. } \\
\text { nih.gov/geo }\end{array}$ & 19,554 datasets \\
\hline ENCODE & $\begin{array}{l}\text { The Encyclopedia of DNA Elements (ENCODE) Consortium: decoding } \\
\text { functional elements in DNA (17). }\end{array}$ & $\begin{array}{l}\text { www.encodeproject. } \\
\text { org }\end{array}$ & $\begin{array}{l}\text { Cancer cell lines } \\
\text { available }\end{array}$ \\
\hline CCLE & $\begin{array}{l}\text { The Cancer Cell Line Encyclopedia (CCLE) project: genomics and } \\
\text { visualization in about 1,000 cell lines. Drug sensitivity available } \\
\text { for the cell lines (78). }\end{array}$ & $\begin{array}{l}\text { www.broadinstitute. } \\
\text { org/ccle/home }\end{array}$ & $\begin{array}{l}\text { Genomic character } \\
\text { ization of } 1,000 \\
\text { cell lines }\end{array}$ \\
\hline PeptideAtlas & An archive of proteome information (21) & $\begin{array}{l}\text { www.peptideatlas. } \\
\text { org }\end{array}$ & 99 datasets \\
\hline PRIDE & $\begin{array}{l}\text { PRoteomics IDEntifications (PRIDE) database: protein and peptide } \\
\text { identifications, post-translational modifications (22). Mass spectrometry } \\
\text { based proteomics data available. }\end{array}$ & $\begin{array}{l}\text { www.ebi.ac.uk/ } \\
\text { pride/archive }\end{array}$ & 290 datasets \\
\hline
\end{tabular}

directed network structures, STRING also has undirected network structures. The hiPathDB (32) introduces a unique concept of "superpathways," that consolidates multiple resources of pathway databases (NCl-Nature PID (34), Reactome (30), BioCarta (35) and KEGG (29)), resulting in the most extensive hierarchical network structures.

\section{COMPUTATIONAL MODELING AND ITS APPLICATION TO CANCER}

Depending on prior knowledge usage, computational modeling, a key component in systems biology frameworks, can be divided into two modeling methods: hybrid method and data-driven method. The former incorporates prior knowledge in model development, while the latter infers networks or hypotheses directly from measurements, without prior knowledge. The tools described below are summarized in Table 2.

\section{DATA-DRIVEN METHODS}

Data-driven methods have been used to correlate mutual information as gene-gene connection for network construction (36-38), resulting in undirected networks. ARACNE (minet. meyerp.com) (39), another widely used free web-based tool, uses mutual information for constructing gene regulatory networks from transcriptome datasets. In principle, starting from all connected entries, ARACNE applies a mutual information data processing inequality (MIDPI) rule to the two adjacent edges for removing non-interacting edges (39). Since its introduction, ARACNE has been widely used in the field of cancer systems biology. Recently, ARACNE was used to describe three hypothetical stages of the epithelial-mesenchymal transition in cancer metastasis (40).

The $\mathrm{R}$ package, weighted gene co-expression network analysis (WGCNA, genetics.ucla.edu/Rpackages/WGCNA) (36), is another network generation tool. Specifically, WGCNA builds gene-gene co-expression networks from all pairwise correlations, among expressed genes, across the entire transcriptome. To infer connections in a network, WGCNA (36) uses a 
Table 2. Summary of tools in network construction. The short description and homepages of some tools in the manuscript are summarized

\begin{tabular}{|c|c|c|}
\hline Class & Name & Homepage and description \\
\hline \multirow[t]{6}{*}{ Data-driven model } & ARACNE (39) & $\begin{array}{l}\text { - http://minet.meyerp.com/ } \\
\text { - Standalone tool available } \\
\text { - Mutual information based network generation }\end{array}$ \\
\hline & WGCNA (36) & $\begin{array}{l}\text { - https://labs.genetics.ucla.edu/horvath/CoexpressionNetwork/Rpackages/WGCNA } \\
\text { - R package available } \\
\text { - Correlation-based network generation }\end{array}$ \\
\hline & Cancer Landscapes (45) & $\begin{array}{l}\text { - http://www.cancerlandscapes.org/ } \\
\text { - Web-based tool } \\
\text { - Sparse inverse covariance selection-based network generation }\end{array}$ \\
\hline & Ultranet $(46,47)$ & $\begin{array}{l}\text { - www.broadinstitute.org/ultranet } \\
\text { - Standalone tool available } \\
\text { - Sparse inverse covariance selection-based network generation }\end{array}$ \\
\hline & Banjo (48) & $\begin{array}{l}\text { - https://users.cs.duke.edu/ amink/software/banjo/ } \\
\text { - Standalone tool available } \\
\text { - Network generation by using Bayesian networks }\end{array}$ \\
\hline & CATNET (50) & $\begin{array}{l}\text { - https://cran.r-project.org/web/packages/catnet/index.html } \\
\text { - Standalone tool available } \\
\text { - Bayesian networks }\end{array}$ \\
\hline \multirow[t]{3}{*}{ Hybrid model } & EDDY (28) & $\begin{array}{l}\text { - http://biocomputing.tgen.org/software/EDDY } \\
\text { - Standalone tool available } \\
\text { - Gene sets and Bayesian networks combined }\end{array}$ \\
\hline & PATHOME (7) & $\begin{array}{l}\text { - Web version of the algorithm under construction (available on request) } \\
\text { - KEGG pathways and correlation-based statistic combined }\end{array}$ \\
\hline & SPIA (58) & $\begin{array}{l}\text { - http://bioconductor.org/packages/release/bioc/html/SPIA.html } \\
\text { - R package available } \\
\text { - KEGG pathways and permutation tests combined }\end{array}$ \\
\hline
\end{tabular}

weighted adjacency matrix between gene pairs by calculating power adjacency function (41), resulting in connections among the gene entries. WGCNA has also been applied to diverse diseases, including cancer, for identifying therapeutic targets and tumorigenesis "driver" genes (42-44).

Despite the great success of correlation- and mutual information-based approaches, these approaches often generate extensive links between network entries. Consequently, methods have now been introduced to reduce non-significant links. For example, sparse inverse covariance selection (SICS) $(45,46)$ infers a gene regulatory network from various data types by reducing non-significant links. The main function of SCIS is to identify a subset of network entries that consists of statistically significant or optimal pairwise correlations, based on the entire correlation (equivalent to covariance) matrix between all the entries. The benefit of subset identification is that it can provide statistically direct relations with smaller number of entries. SICS methods aim at maximizing or optimizing log-likelihood of pairwise correlations, assuming pairwise correlations as Gaussian graphical models $(46,47)$ or multivariate Gaussian models (45). Cancer Landscapes (cancerlandscapes.org) utilizes SICS, not only to provide multiple cancer network modules, but also to integrate multilevel omics data types into statistical network modules (45).

Unlike ARACNE and WGCNA, there are several approaches to generate directed networks (Fig. 1C). Bayesian networks, another data-driven approach, utilizes a basic conditional independence (48-51). Bayesian networks is, by definition, that joint density probability of biological entries (e.g., genes) is the product of conditional probabilities of the entries in the omics data $(38,52)$. The definition naturally confers the ability to prune edges of the conditionally independent entries. Also, conditional dependency defines statistically casual relationships among gene entries, resulting in directed networks. The purpose of Bayesian networks is to identify the set of conditional probabilities that best describe measurements (e.g., gene expression) of biological entries in omics databases.

Banjo (users.cs.duke.edu/software) is another gene regulatory network generation tool that utilizes Bayesian network frameworks, resulting in directed networks (48). Banjo is applicable not only for single-state transcriptome data, but also for time-series data. Banjo (Bayesian network interference with Java objects) uses the multiple types of heuristic network searching to find candidate networks (equivalently, graphs), such as simulated annealing with a greedy algorithm (53), and genetic algorithm (48). The conditional probability densities of each network are estimated, and the network scores (e.g., Bayesian Information Criterion (BIC), Bayesian Dirichlet equivalence (BDe)) are then calculated. Finally, Banjo reports the network with the best score, based on its best directed edges between its entries. Banjo has also been applied to leukemia, revealing miRNA-relating network hierarchy by 
merging gene expression, gene regulatory networks, and copy number alterations (54).

One obstacle to all these prediction methods is that there are no "gold standards" for data-driven network generation tools. Consequently, the performance of the data-driven methods depends on data types, model parameter settings, network size, and network topology (55).

\section{HYBRID METHODS}

In hybrid methods, models are generated to analyze highthroughput data via prior knowledge (e.g., gene sets, pathways) (56), resulting in network inference. Traditionally to date, hybrid methods use pathways as prior knowledge. Recently, gene sets have been recognized as another prior knowledge source for inferring networks that consist of entries and their mutual interactions.

Another tool, EDDY (evaluation of dependency differentiality) (28) considers two conditions, and applies Bayesian networks framework to all the gene sets. EDDY selects the best network structure for each gene set, by using Jensen-Shannon (IS) divergences and permutation tests from all possible network structures. The tool then calculates the two probability density distributions of a network structure for the two conditions. Subsequently, EDDY calculates JS divergence for the two distributions of the network structure, measuring JS divergence as the difference of the two distributions. The significance of JS divergence is measured by the permutation test, identifying the best network structure having statistically significant JS divergence. The output is a network that consists of the entries (of the gene set) and their interactions between the entries. The tool was recently applied to glioblastoma multiforme (GBM), resulting in the successful identification of specific molecular subtypes of glioblastoma (28).

Prior pathway information with omics data has been incorporated into statistical frameworks for the past ten years $(7,8,57)$, successfully generating network structures. In this approach, the challenge to build the statistical framework is developing and defining a statistic reflecting pathway topology. Pathway topology indicates interaction types (e.g., activation, inhibition, modification) as well as order (e.g., upstream, downstream) of biological entries. Another tool, SPIA (signaling pathway impact analysis) (58) (bioconductor.org/packages/ release/bioc/html/SPIA.html), utilizes the KEGG pathway database as prior knowledge. Instead of utilizing the individual signaling molecules (in KEGG pathways), SPIA aligns the consecutive KEGG signaling "flows" with omics data. Additionally, SPIA now considers two types of a flow between two adjacent signaling molecules: activation and inhibition. SPIA quantitatively measures influence (i.e., perturbation statistic in a given pathway) on signal cascading flows by using omics data between two experimental groups. For any given pathway, SPIA obtains $P$ values for the perturbation statistic by using permutation tests. SPIA also reconstructs statistically significant pathways in a network. Recently, SPIA was applied to aggressive prostate cancer, discovering that the disease shares a pathway network with small cell lung cancer (59).

We also developed pathway topology-driven hybrid methods $(7,8)$, specifically for network generation, including PATHOME (7). These two methods also input the KEGG database (29) as prior knowledge for network generation. The earlier algorithm (8) (henceforth, pre-PATHOME) identified subsets of all KEGG pathways by utilizing permutation-oriented statistical tests, based on a whole transcriptome. Since graphical structures of the KEGG pathways are too complex, we decomposed to all the possible paths $(\sim 130$ million, equivalently, subpathways $)$ by traversing the graph structures.

In pre-PATHOME, each path consists of biological entries and their mutual interactions between adjacent two entries, either activation or inhibition. Given a subpathway, we devised a statistic to consider interactions (equivalently, edges) of two adjacent entries, as well as orders of biological entities (8). We assumed the first order Markov property (denoted as $F_{\text {edge }}$ in (8)) where the fold-changes of the entities were regarded as observations. Subsequently, we performed permutationbased statistical tests for the product of $\mathrm{F}_{\text {edge }}$ and two additional statistics in each path. The statistically significant paths were collected and visualized. The pre-PATHOME was applied to an early onset colorectal cancer (CRC) dataset (60), revealing the pathways of epithelial-to-mesenchymal transition and immunosuppression even in normal adjacent cells of the CRC patients (8). The pre-PATHOME (8) was also deployed to identify trastuzumab-resistance pathways relating to networks in HER2(+) breast cancer (61), revealing five biomarker candidates associated with trastuzumab non-responsiveness (ATF4, CHEK2, ENAH, ICOSLG, and RAD51).

Our group recently developed another hybrid method, PATHOME, (7). The pre-PATHOME (8) assumed that all interactions in a subpathway are dependent on their upstream entities (the so called, first order Markov property). PATHOME assumes that all edges in a subpathway are independent, adopting a two-stage strategy in our statistical framework (7). In the first stage, out of 130 million KEGG subpathways, PATHOME selects those with their edges aligned with correlations. In the second stage, we test the selected subpathways under the null hypothesis, that no differential correlation patterns between two groups are observed. Despite the independence assumption among edges, PATHOME showed better agreement with a cancer signaling reference set (62), when compared to other gene set analysis tools (e.g., DAVID (63), and GSEA (25)).

PATHOME has also been applied for delineating druggable target candidates, as well as molecular mechanisms, in both gastric and breast cancers $(7,64,65)$. Recently, PATHOME was applied to gastric cancer (GC) transcriptome datasets, suggesting a HNF4 $\alpha$ WNT5A axis to be a new druggable signaling, as well as having a clinical relevance in diffuse type GC $(64,65)$. Since trastuzumab treatment of HER2-positive GC 
tumors show limited benefit, compared with ERBB2-positive breast cancer (66), PATHOME was applied to high ERBB2 (equivalently, HER2)- expressing GC patient datasets in the TCGA $(64,67)$. In these analyses, PATHOME revealed that NFBIE, PTK2, and PIK3CA, all downstream molecules of ERBB2, associate with genomic characteristics of high ERBB2expressing GC patients over low ERBB2-expressing GC patients (64).

\section{CONCLUSIONS}

Systems biology is a general modeling framework that utilizes high-throughput data and prior knowledge, to result in network inference and hypotheses suggestions. Most network generation tools are based on whole transcriptome data. Using statistical models, the integration of other data types into network topology is still challenging. For example, for effective targeted therapy, the effects of mutations need to be incorporated into pathway topology under the systems biology frameworks (68). Also, for facilitation of translating cancer big data toward therapeutic benefit, pharmacokinetics/pharmacodynamics assessments (69-71) need to be considered in network generation in future.

Although this review does not describe visualization tools, intuitive and informative graphical visualization of the models should keep pace with systems biology tools (72-74).

\section{ACKNOWLEDGEMENTS}

This work was supported by the Gachon University Gil Medical Center (Grant number: 2016-06), and performed by a subproject of KISTI (Korea Institute of Science and Technology Information)'s project No. P16018 (Development of HPCbased Big Data for healthy Aging Society) funded by (Ministry of Science, ICT, and Future Planning). The Author thanks Curt Balch for editing the manuscript.

\section{CONFLICTS OF INTEREST}

The authors have no conflicting financial interests.

\section{REFERENCES}

1. Werner HM, Mills GB and Ram PT (2014) Cancer Systems Biology: a peek into the future of patient care? Nat Rev Clin Oncol 11, 167-176

2. Soon WW, Hariharan M and Snyder MP (2013) Highthroughput sequencing for biology and medicine. Mol Syst Biol 9, 640

3. Chuang HY, Hofree M and Ideker T (2010) A decade of systems biology. Annu Rev Cell Dev Biol 26, 721-744

4. Jost D, Nowojewski A and Levine E (2011) Small RNA biology is systems biology. BMB Rep 44, 11-21

5. Nam S, Long X, Kwon C, Kim S and Nephew KP (2012) An integrative analysis of cellular contexts, miRNAs and
mRNAs reveals network clusters associated with antiestrogen-resistant breast cancer cells. BMC Genomics 13,732

6. Rho S, You S, Kim Y and Hwang D (2008) From proteomics toward systems biology: integration of different types of proteomics data into network models. BMB Rep 41, 184-193

7. Nam S, Chang HR, Kim KT et al (2014) PATHOME: an algorithm for accurately detecting differentially expressed subpathways. Oncogene 33, 4941-4951

8. Nam S and Park T (2012) Pathway-based evaluation in early onset colorectal cancer suggests focal adhesion and immunosuppression along with epithelial-mesenchymal transition. PLoS One 7, e31685

9. Altaf-UI-Amin M, Afendi FM, Kiboi SK and Kanaya S (2014) Systems biology in the context of big data and networks. Biomed Res Int 2014, 428570

10. Marx V (2013) Drilling into big cancer-genome data. Nat Meth 10, 293-297

11. Cancer Genome Atlas Research Network, Weinstein JN, Collisson EA et al (2013) The Cancer Genome Atlas Pan-Cancer analysis project. Nat Genet 45, 1113-1120

12. Zhang J, Baran J, Cros A et al (2011) International Cancer Genome Consortium Data Portal-a one-stop shop for cancer genomics data. Database (Oxford) 2011, bar026

13. Ghosh S, Matsuoka Y, Asai Y, Hsin KY and Kitano H (2011) Software for systems biology: from tools to integrated platforms. Nat Rev Genet 12, 821-832

14. Zierer J, Menni C, Kastenmuller G and Spector TD (2015) Integration of 'omics' data in aging research: from biomarkers to systems biology. Aging Cell 14, 933-944

15. Pecina-Slaus $N$ and Pecina $M$ (2015) Only one health, and so many omics. Cancer Cell Int 15, 64

16. International Cancer Genome Consortium, Hudson TJ, Anderson W et al (2010) International network of cancer genome projects. Nature 464, 993-998

17. Encode Project Consortium (2012) An integrated encyclopedia of DNA elements in the human genome. Nature 489, 57-74

18. Tryka KA, Hao L, Sturcke A et al (2014) NCBI's Database of Genotypes and Phenotypes: dbGaP. Nucleic Acids Res 42, D975-979

19. Barrett T, Wilhite SE, Ledoux $P$ et al (2013) NCBI GEO: archive for functional genomics data sets-update. Nucleic Acids Res 41, D991-995

20. Rocca-Serra P, Brazma A, Parkinson H et al (2003) ArrayExpress: a public database of gene expression data at EBI. C R Biol 326, 1075-1078

21. Kusebauch U, Deutsch EW, Campbell DS, Sun Z, Farrah T and Moritz RL (2014) Using PeptideAtlas, SRMAtlas, and PASSEL: Comprehensive Resources for Discovery and Targeted Proteomics. Curr Protoc Bioinformatics 46, 13.25.1-13.25.28

22. Jones P and Cote R (2008) The PRIDE proteomics identifications database: data submission, query, and dataset comparison. Methods Mol Biol 484, 287-303

23. Liberzon A, Birger C, Thorvaldsdottir H, Ghandi $M$, Mesirov JP and Tamayo P (2015) The Molecular Signatures Database (MSigDB) hallmark gene set collection. Cell Syst $1,417-425$ 
24. Nam S, Li M, Choi K, Balch C, Kim S and Nephew KP (2009) MicroRNA and mRNA integrated analysis (MMIA): a web tool for examining biological functions of microRNA expression. Nucleic Acids Res 37, W356-362

25. Subramanian A, Tamayo P, Mootha VK et al (2005) Gene set enrichment analysis: a knowledge-based approach for interpreting genome-wide expression profiles. Proc Natl Acad Sci U S A 102, 15545-15550

26. Maciejewski H (2014) Gene set analysis methods: statistical models and methodological differences. Brief Bioinform 15, 504-518

27. Emmert-Streib F, Tripathi S and de Matos Simoes R (2012) Harnessing the complexity of gene expression data from cancer: from single gene to structural pathway methods. Biol Direct 7, 44

28. Jung S and Kim S (2014) EDDY: a novel statistical gene set test method to detect differential genetic dependencies. Nucleic Acids Res 42, e60

29. Kanehisa M and Goto S (2000) KEGG: kyoto encyclopedia of genes and genomes. Nucleic Acids Res 28, 27-30

30. Croft D, Mundo AF, Haw R et al (2014) The Reactome pathway knowledgebase. Nucleic Acids Res 42, D472477

31. Szklarczyk D, Franceschini A, Wyder S et al (2015) STRING v10: protein-protein interaction networks, integrated over the tree of life. Nucleic Acids Res 43, D447-452

32. Yu N, Seo J, Rho K et al (2012) hiPathDB: a humanintegrated pathway database with facile visualization. Nucleic Acids Res 40, D797-802

33. Hucka M, Finney A, Sauro HM et al (2003) The systems biology markup language (SBML): a medium for representation and exchange of biochemical network models. Bioinformatics 19, 524-531

34. Schaefer CF, Anthony K, Krupa S et al (2009) PID: the Pathway Interaction Database. Nucleic Acids Res 37, D674-679

35. Nishimura D (2001) BioCarta. Biotech Software \& Internet Report 2, 117-120

36. Allen JD, Xie Y, Chen M, Girard L and Xiao G (2012) Comparing statistical methods for constructing large scale gene networks. PLoS One 7, e29348

37. Butte AJ and Kohane IS (2000) Mutual information relevance networks: functional genomic clustering using pairwise entropy measurements. Pac Symp Biocomput, 418-429

38. Markowetz F and Spang R (2007) Inferring cellular networks-a review. BMC Bioinformatics 8 Suppl 6, S5

39. Margolin AA, Nemenman I, Basso K et al (2006) ARACNE: an algorithm for the reconstruction of gene regulatory networks in a mammalian cellular context. BMC Bioinformatics 7 Suppl 1, S7

40. Tanaka H and Ogishima S (2015) Network biology approach to epithelial-mesenchymal transition in cancer metastasis: three stage theory. J Mol Cell Biol 7, 253-266

41. Zhang B and Horvath S (2005) A general framework for weighted gene co-expression network analysis. Stat Appl Genet Mol Biol 4, 12287

42. Bailey P, Chang DK, Nones K et al (2016) Genomic analyses identify molecular subtypes of pancreatic cancer.
Nature 531, 47-52

43. Gnad F, Doll S, Manning G, Arnott D and Zhang Z (2015) Bioinformatics analysis of thousands of TCGA tumors to determine the involvement of epigenetic regulators in human cancer. BMC Genomics 16 Suppl 8, S5

44. Horvath S, Zhang B, Carlson M et al (2006) Analysis of oncogenic signaling networks in glioblastoma identifies ASPM as a molecular target. Proc Natl Acad Sci U S A 103, 17402-17407

45. Kling T, Johansson P, Sanchez J, Marinescu VD, Jornsten $R$ and Nelander S (2015) Efficient exploration of pancancer networks by generalized covariance selection and interactive web content. Nucleic Acids Res 43, e98

46. Jarvstrat L, Johansson M, Gullberg U and Nilsson B (2013) Ultranet: efficient solver for the sparse inverse covariance selection problem in gene network modeling. Bioinformatics 29, 511-512

47. Storry JR, Joud M, Christophersen MK et al (2013) Homozygosity for a null allele of SMIM1 defines the Vel-negative blood group phenotype. Nat Genet 45, 537-541

48. Yu J, Smith VA, Wang PP, Hartemink AJ and Jarvis ED (2004) Advances to Bayesian network inference for generating causal networks from observational biological data. Bioinformatics 20, 3594-3603

49. Frolova A and Wilczyński B (2015) Distributed Bayesian Networks Reconstruction on the Whole Genome Scale. bioRxiv doi: 10.1101/016683

50. Salzman P and Almudevar A (2006) Using complexity for the estimation of Bayesian networks. Stat Appl Genet Mol Biol 5, Article21

51. Chen X, Chen $M$ and Ning K (2006) BNArray: an R package for constructing gene regulatory networks from microarray data by using Bayesian network. Bioinformatics 22, 2952-2954

52. Bansal $M$, Belcastro $V$, Ambesi-Impiombato $A$ and $d i$ Bernardo D (2007) How to infer gene networks from expression profiles. Mol Syst Biol 3, 78

53. Adabor ES, Acquaah-Mensah GK and Oduro FT (2015) SAGA: a hybrid search algorithm for Bayesian Network structure learning of transcriptional regulatory networks. J Biomed Inform 53, 27-35

54. Volinia S, Galasso M, Costinean S et al (2010) Reprogramming of miRNA networks in cancer and leukemia. Genome Res 20, 589-599

55. Madhamshettiwar PB, Maetschke SR, Davis MJ, Reverter A and Ragan MA (2012) Gene regulatory network inference: evaluation and application to ovarian cancer allows the prioritization of drug targets. Genome Med 4, 41

56. Galvanauskas V, Simutis R and Lubbert A (2004) Hybrid process models for process optimisation, monitoring and control. Bioprocess Biosyst Eng 26, 393-400

57. Khatri P, Sirota M and Butte AJ (2012) Ten years of pathway analysis: current approaches and outstanding challenges. PLoS Comput Biol 8, e1002375

58. Tarca AL, Draghici S, Khatri P et al (2009) A novel signaling pathway impact analysis. Bioinformatics 25, 75-82

59. Smith BA, Sokolov A, Uzunangelov V et al (2015) A basal 
stem cell signature identifies aggressive prostate cancer phenotypes. Proc Natl Acad Sci U S A 112, E6544-6552

60. Hong Y, Ho KS, Eu KW and Cheah PY (2007) A susceptibility gene set for early onset colorectal cancer that integrates diverse signaling pathways: implication for tumorigenesis. Clin Cancer Res 13, 1107-1114

61. Nam S, Chang HR, Jung HR et al (2015) A pathway-based approach for identifying biomarkers of tumor progression to trastuzumab-resistant breast cancer. Cancer Lett 356, 880-890

62. Vogelstein B and Kinzler KW (2004) Cancer genes and the pathways they control. Nat Med 10, 789-799

63. Huang da W, Sherman BT and Lempicki RA (2009) Systematic and integrative analysis of large gene lists using DAVID bioinformatics resources. Nat Protoc 4, 44-57

64. Chang HR, Nam S, Kook MC et al (2016) HNF4alpha is a therapeutic target that links AMPK to WNT signalling in early-stage gastric cancer. Gut 65, 19-32

65. Chang HR, Park HS, Ahn YZ et al (2016) Improving gastric cancer preclinical studies using diverse in vitro and in vivo model systems. BMC Cancer 16, 200

66. Bang YJ, Van Cutsem E, Feyereislova A et al (2010) Trastuzumab in combination with chemotherapy versus chemotherapy alone for treatment of HER2-positive advanced gastric or gastro-oesophageal junction cancer (ToGA): a phase 3, open-label, randomised controlled trial. Lancet 376, 687-697

67. Cancer Genome Atlas Research Network (2014) Comprehensive molecular characterization of gastric adenocarcinoma. Nature 513, 202-209

68. Hernansaiz-Ballesteros RD, Salavert F, Sebastian-Leon P, Aleman A, Medina I and Dopazo J (2015) Assessing the impact of mutations found in next generation sequencing data over human signaling pathways. Nucleic Acids Res 43, W270-275

69. Griffith M, Griffith OL, Coffman AC et al (2013) DGldb: mining the druggable genome. Nat Methods 10, 12091210

70. Wishart DS, Knox C, Guo AC et al (2006) DrugBank: a comprehensive resource for in silico drug discovery and exploration. Nucleic Acids Res 34, D668-672

71. Whirl-Carrillo M, McDonagh EM, Hebert JM et al (2012) Pharmacogenomics knowledge for personalized medicine. Clin Pharmacol Ther 92, 414-417

72. Franz M, Lopes CT, Huck G, Dong Y, Sumer O and Bader GD (2016) Cytoscape.js: a graph theory library for visualisation and analysis. Bioinformatics 32, 309-311

73. Jang Y, Yu N, Seo J, Kim S and Lee S (2016) MONGKIE: an integrated tool for network analysis and visualization for multi-omics data. Biol Direct 11, 10

74. Smoot ME, Ono K, Ruscheinski J, Wang PL and Ideker T (2011) Cytoscape 2.8: new features for data integration and network visualization. Bioinformatics 27, 431-432

75. Cerami E, Gao J, Dogrusoz U et al (2012) The cBio cancer genomics portal: an open platform for exploring multidimensional cancer genomics data. Cancer Discov 2, 401404

76. Parkinson H, Sarkans U, Kolesnikov N et al (2011) ArrayExpress update--an archive of microarray and highthroughput sequencing-based functional genomics experiments. Nucleic Acids Res 39, D1002-1004

77. Zhu J, Sanborn JZ, Benz S et al (2009) The UCSC Cancer Genomics Browser. Nat Methods 6, 239-240

78. Barretina J, Caponigro G, Stransky N et al (2012) The Cancer Cell Line Encyclopedia enables predictive modelling of anticancer drug sensitivity. Nature 483, 603-607 